\title{
ORIGINAL
}

\section{EVALUACIÓN DE LA GESTIÓN HOSPITALARIA DE RESIDUOS SANITARIOS EN EL PRINCIPADO DE ASTURIAS}

\author{
Susana Llorente Álvarez, Pedro Arcos González y Rafael González Estrada. \\ Servicio de Inspección de Salud. Consejería de Servicios Sociales del Principado de Asturias.
}

\section{RESUMEN}

Fundamento: Con el objetivo de evaluar la gestión de los residuos sanitarios de los 12 hospitales asturianos con financiación pública. se visitaron los servicios de ayuellos hospitales que tienen especial relevancia en la generación de residuos (91 servicios en total). Los hospitales evaluados representan el $75.10 \%$ del total de camas hospitalarias del Principado.

Métodos: Se realizó una entrevista al personal de los 91 servicios, siempre por el mismo entrevistador, y protocolizada según los criterios de la Joint Commission on Accreditation of Health Care Organizations. La información obtenida fue luego verificada por el entrevistador mediante la observación directa.

Resultados: Entre los resultados más relevantes destaca la ausencia de envases adecuados para los residuos de tipo «clínico» en el $28.5 \%$ de los servicios visitados. Asimismo, el 82.4\% de los servicios clasifican incorrectamente este tipo de residuos. El porcentaje de clasificación incorrecta es del $1.1 \%$ para los residuos «especiales». En los hospitales grandes (más de 300 camas), el $60 \%$ de los servicios disponen de información sobre el manejo de los residuos. pero sólo en un $40 \%$ de estos hospitales se supervisa adecuadamente la gestión del residuo por parte del Servicio de Medicina Preventiva u otro servicio equivalente.

Conclusiones: El grado de incorrecta clasificación del residuo sanitario que se ha hallado sugiere que, para mejorar la gestión intrahospitalaria del residuo, es preciso aumentar el grado de información, modificar determinadas actitudes del personal hospitalario. y reforzar el nivel de supervisión ejercido por los Servicios de Medicina Preventiva.

Palabras clave: Residuos sanitarios. Evaluación.Hospital.Prevención. Personal sanitario.

\section{ABSTRACT \\ Evaluation of the Hospital Management of Sanitary Waste in the Principality of Asturias}

Background: With the object of evaluating the management of sanitary waste of 12 publicly funded Asturian hospitals, a visit was paid to the hospital services, particularly significant as regards the generation of waste (a total of 91 services). The evaluated hospitals represent $75,10 \%$ of the total hospital beds in the Principality.

Methods: Interviews with personnel from 91 different services were undertaken, always by the same interviewer, and according to the rules of the Joint Commission of Health Care Organizations. The information obtained was afterwards verified by the interviewer by means of direct observation.

Results: Amongst the most relevant results, the absence of adequate containers for «clinical» waste in $28,5 \%$ of the services visited, stands out. Into the bargain. $82,4 \%$ of the services classify this type of waste incorrectly. The percentage of incorrect classification is $1,1 \%$ for «special» waste. In large hospitals (with over 300 beds), $60 \%$ of the services have information on the handling of waste, but only $40 \%$ of these hospitals have adequate supervision over the management of waste by the Service of Preventive Medicine or another equivalent service.

Conclusions: The level of incorrect classification of sanitary waste which has been discovered suggests that, in order to improve the intra-hospital management of waste, it is necessary to increase the amount of information, modify certain attitudes of the hospital staff and reinforce the level of supervision executed by the Services of Preventive Medicine.

Key words: Sanitary Waste. Evaluation. Hospital. Prevention. Health professionals.
Correspondencia:

Pedro Arcos González

Servicio de Inspección de Salud

Consejería de Servicios Sociales

General Elorza, 32

33001 Oviedo

Fax: (98) 5106520

\section{INTRODUCCIÓN}

Aunque el interés sanitario y medioambiental de los residuos hospitalarios no es nuevo, fue a partir de los años 80 , y especialmente 
tras la aparición del Sida, cuando el tema de los residuos producidos por los hospitales comenzó a ser percibido como un problema potencial para la salud pública ${ }^{1-4}$. A este interés ha contribuido también el progresivo endurecimiento de las regulaciones medioambientales, inicialmente en determinados países (Francia, Alemania, Holanda) y, posteriormente, en el conjunto de los estados miembros de la Unión Europea ${ }^{6-8}$.

En 1989 la Administración del Principado de Asturias inició la evaluación de la gestión de los residuos en los centros sani$\operatorname{tarios}^{9,10}$ para poner en marcha un Plan Integral de Residuos Sanitarios. En este sentido, dos de los factores que fueron identificados como limitantes de una buena gestión de estos residuos eran la ausencia de una norma legal estatal específica (aunque determinadas Comunidades Autónomas han regulado el tema en sus territorios) ${ }^{1-13}$, así como la ausencia de criterios homogéneos de definición, clasificación, envasado, transporte y eliminación de los diferentes tipos de residuos ${ }^{14}$. También aparecieron como circunstancias limitantes la inadecuación estructural de los hospitales, la ausencia de regulación interna y el escaso cono- cimiento del personal sanitario y no sanitario en relación al problema ${ }^{15,16}$.

La Figura 1 muestra la producción de residuos hospitalarios tipo III (aquellos compuestos de material patológico o infeccioso capaz de producir contagio o toxicidad y que han de ser sometidos a un tratamiento de eliminación específico) en las principales Áreas de Salud de Asturias. En 1995 los hospitales asturianos produjeron una media de 212 gramos de residuo tipo III por cama y día (desviación estándar de 150 gramos $)^{17}$. La gran variabilidad observada en esta distribución de la producción, en hospitales de características similares, nos induce a considerar que existen diferencias importantes en la gestión intrahospitalaria del residuo. Por otro lado, una gestión inadecuada implica la existencia de mayores riesgos asociados al manejo del residuo, así como un aumento en los costes de tratamiento y eliminación ${ }^{18,19}$. Dada la relevancia del tema, se planteó este estudio con el objetivo de evaluar la gestión hospitalaria de los residuos sanitarios en los hospitales asturianos de financiación pública, así como identificar los factores limitantes internos para una gestión adecuada.

Figura 1

Producción anual de residuos hospitalarios en Asturias por áreas de salud en 1995

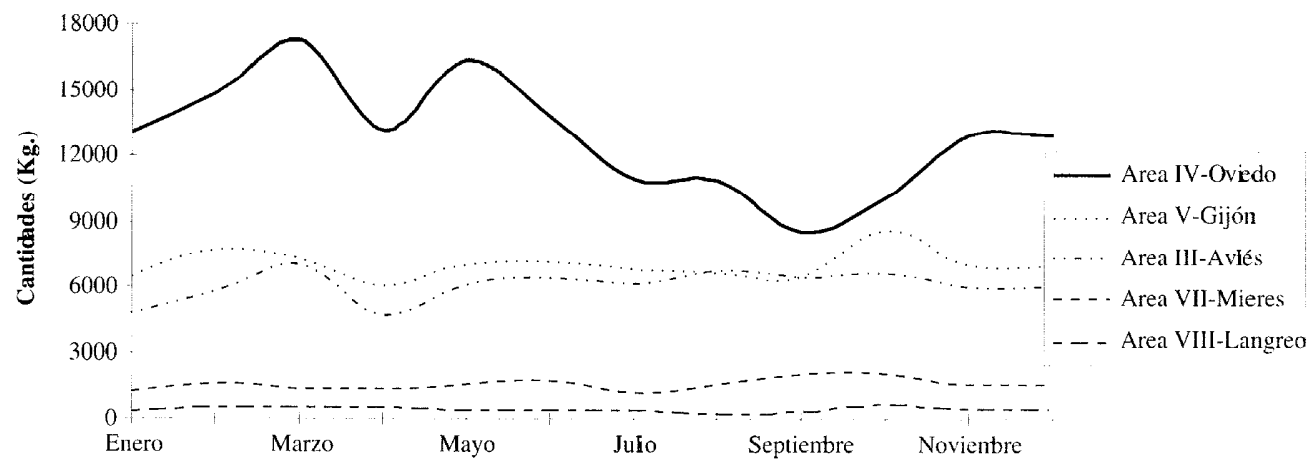




\section{MATERIAL Y MÉTODOS}

El estudio se realizó examinando la información obtenida en los 12 hospitales del Principado de Asturias que tienen financiación pública ( 9 del Insalud y 3 concertados) y cuyas características se resumen en la Tabla 1 . En conjunto, estos 12 hospitales representan el $75,10 \%$ del total de las camas hospitalarias del Principado ${ }^{20}$.

El trabajo de campo, realizado entre agosto y octubre de 1995, consistió en visitar las unidades o servicios que tenían especial relevancia, cuantitativa y/o cualitativa, en lo que se refiere a la generación de residuos. El total incluyó 10 servicios de urgencias, $10 \mathrm{dc}$ anatomía patológica, 11 laboratorios de hematología, bioquímica y microbiología, 7 unidades de hemodiálisis, 8 unidades de infecciosas, 12 plantas de hospitalizaciòn (aunque hay varias por centro, se tomó como muestra una por cada hospital pues el funcionamiento es similar para todas ellas en cada centro) y 11 áreas quirúrgicas (zona de quirófanos y salas dedicadas a cirugía menor ambulatoria y/o técnicas invasivas). En estos servicios se revisaron todos los aspectos relativos a la recogida y circulación de residuos dentro de la unidad, y hasta su almacenamiento definitivo en el depósito general de residuos del hospital.

La información fue recogida mediante una entrevista protocolizada con el personal de cada unidad, responsables de enfermería, servicio de Medicina Preventiva o equivalente y servicios de Mantenimiento y Limpieza. En todos los casos la información fue verificada con la observación directa en cada unidad llevada a cabo siempre por la misma persona.

Una vez recogida la información, el entrevistador cumplimentó dos cuestionarios con respuestas cerradas y excluyentes: el primero referido a cada unidad o servicio y el segundo para el conjunto del hospital (Anexos 1 y 2). Para elaborar el cuestionario se usaron el manual de Gestión de los Residuos Sanitarios en el Principado de Asturias ${ }^{21}$, así como los criterios establecidos por la Joint Commission on Accreditation of Health Care Organizations de Estados Unidos ${ }^{22}$.

Los criterios y definiciones empleados en el estudio fueron los siguientes. Se consideró dotación adecuada de envases, ubicación, periodicidad de recogida, etc, aquella que cumpliera las características descritas en la

Tabla 1

Descripción de los hospitales incluidos en el estudio

\begin{tabular}{|c|c|c|c|c|}
\hline Centro & $N .^{\circ}$ camas & Asistencia & Titularidad & Concierto \\
\hline COMPLEJO HOSPITALARIO (OVIEDO) & 1.876 & general & insalud & \\
\hline \multicolumn{5}{|l|}{ Hospital Nuestra Señora de Covadonga } \\
\hline \multicolumn{5}{|l|}{ Instituto Nacional de Silicosis } \\
\hline \multicolumn{5}{|l|}{ Hospital General de Asturias } \\
\hline Hospital de Cabueñes (Gijón) & 453 & general & insalud & \\
\hline Hospital de San Agustín (Avilés) & 303 & general & insalud & \\
\hline Hospital Valle del Nalón (Riaño-Langreo) & 268 & general & insalud & \\
\hline Hospital de la Caridad -Jove- (Gijón) & 233 & general & $\begin{array}{l}\text { fundación benéfica } \\
\text { privada }\end{array}$ & si \\
\hline Hospital Álvarez-Buylla (Mieres) & 228 & general & insalud & \\
\hline Hospilal Cruz Roja (Gijón) & 147 & quirúrgico/geriátrico & cruz roja española & $\mathrm{si}$ \\
\hline $\begin{array}{l}\text { Hospital Cármen y Severo Ochoa (Cangas do } \\
\text { Narcea) }\end{array}$ & 134 & general & insalud & \\
\hline Hospital Comarcal de Jarrio (Coaña) & 112 & general & insalud & \\
\hline Hospital de Caridad (Avilés) & 99 & quirúrgico/geriátrico & patronato municipal & $\mathrm{si}$ \\
\hline
\end{tabular}


citada publicación ${ }^{21}$. Asimismo, se consideró separación selectiva adecuada de los residuos aquella basada en la clasificación siguiente que, por otro lado, es la más generalmente aceptada ${ }^{9,11.13,23}$ : a) Residuos tipo I (asimilables a urbanos), serían los no específicamente sanitarios, similares a los que se producen en un domicilio, es decir procedentes de actividades de cocina, limpieza, administración, jardinería, etc. b) Residuos tipo II (clínicos), son los procedentes de la actividad sanitaria asistencial (curas, análisis clínicos, intervenciones quirúrgicas) que no tengan capacidad de producir contagio o toxicidad y; c) Residuos tipo III (especiales), serían aquellos materiales patológicos o infecciosos procedentes de laboratorios de anatomía patológica, microbiología, hematología y bioquímica, restos humanos procedentes de paritorios y quirófanos, que tienen capacidad potencial para producir contagio $y / 0$ toxicidad. Se dividen en traumáticos (punzantes y cortantes) y no traumáticos.

Se consideraron envases adecuados los siguientes: bolsas negras de 69 galgas para los residuos tipo I y bolsas verdes de 69 galgas para los residuos tipo II. Para los residuos tipo III (traumáticos), recipientes amarillos marcados con el anagrama internacional de Bioriesgo, rígidos o semirígidos que permitan la libre sustentación y resistan la perforación interna y externa; dotados de cierre hermético. Para residuos tipo III (no traumáticos) envases de un solo uso, no compactables ni triturables en ningún caso y que pueden ser bolsas amarillas de 200 galgas, de capacidad no superior a 60 litros o doble bolsa amarilla de 400 galgas (si no va a introducirse posteriormente en envase rígido, o envase de 30 a 60 litros de capacidad de material resistente a la humedad, estanco e inviolable).

Además, todos los envases de residuos (tipo I, II y III) estarán constituidos por material opaco, impermeable, con resistencia a la humedad y a la carga estática y que no genere emisiones tóxicas durante su combustión.

Se entendió como periodicidad de recogida adecuada la evacuación de los residuos, una vez llenos los envases, desde el lugar de producción y/o depósito intermedio y al menos cada 24 horas. Hemos considerado transporte correcto por la unidad a aquel realizado en condiciones que no impliquen un riesgo para el personal encargado. El depósito intermedio, dependencia en que se almacenan los residuos hasta su transporte al depósito general del centro, debía tener una ubicación próxima a los lugares de origen del residuo, ser de fácil limpieza y ventilación y disponer de aparato sanitario. El depósito general de residuos debía ser un local de uso exclusivo, ubicado en el límite externo del hospital y dotado de un sistema de ventila ción adecuado, toma de agua, sumidero y detector de incendios, con desinfección diaria y en el que los residuos no permanecieran más de 48 horas. Finalmente, entendimos como circuitos diferenciados (espacial y temporalmente) aquellos que empleaban montacargas específicos para transporte de residuos, o ascensores de uso general inutilizados para otras funciones mientras este se llevaba a cabo y realizado en horario que no interfiriese con la actividad normal del hospital.

\section{RESULTADOS}

En las figuras 2 y 3 se muestran los resultados obtenidos sobre los 91 servicios visitados en los 12 hospitales, así como la información desagregada según el tamaño del hospital, considerando por un lado los hospitales «grandes» (de más de 300 camas), que son los hospitales de referencia para las Áreas Sanitarias III (Avilés), IV (Oviedo) y V (Gijón), y por otro, los hospitales «pequeños» (menos de 300 camas) que son hospitales comarcales o entidades privadas concertadas. 
Figura 2

Porcentaje de servicios que carecen de envases adecuados para cada categoría de residuos producidos

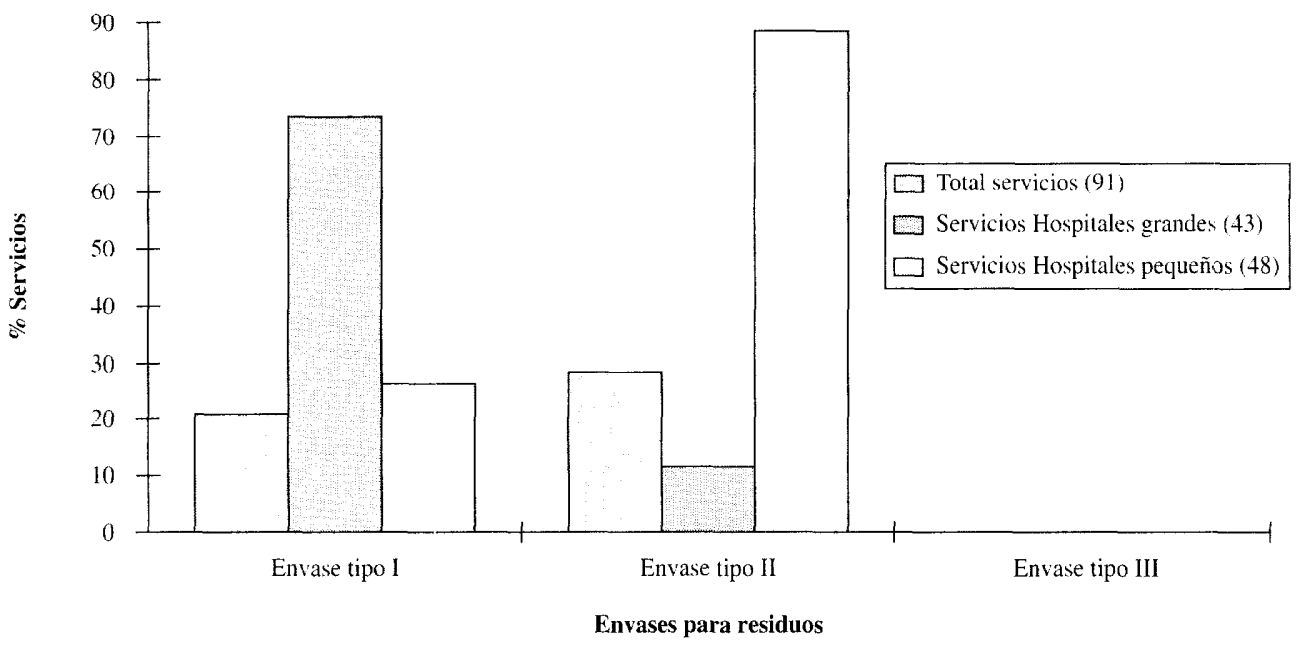

Figura 3

Porcentaje de servicios que clasifican incorrectamente cada tipo de residuos

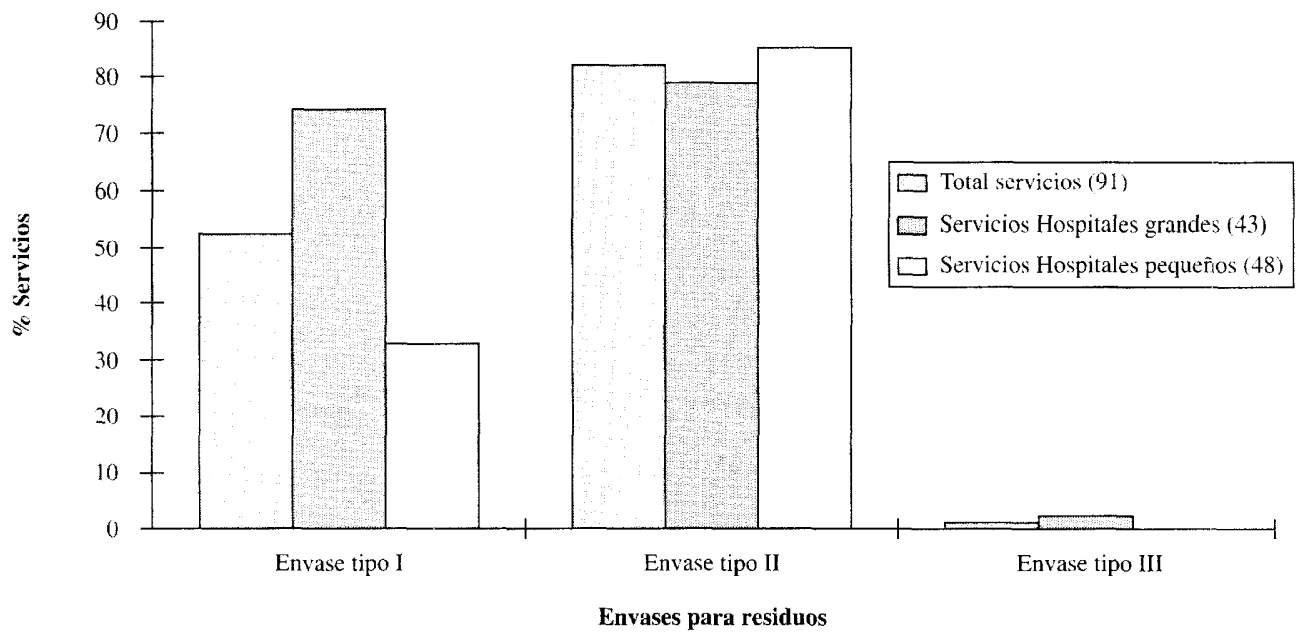


Los dos hallazgos más significativos se refieren a la incorrecta clasificación del residuo sanitario en los diferentes servicios hospitalarios y a la ausencia de envases adecuados a cada tipo de residuo. Respecto a los envases para residuos tipo I, su ausencia se detectó en el $20,8 \%$ del total de servicios visitados. Para este tipo de residuo, los servicios más deficitarios son los de los hospitales grandes (en los que el 73,5\% de los servicios no disponen del envase adecuado). La diferencia en la disponibilidad de este envase entre los hospitales grandes y los pequeños es estadísticamente significativa $\left(\mathrm{chi}^{2}=5.46, \mathrm{p}<0.01\right)$.

Para el conjunto de los hospitales, el $28,5 \%$ de sus servicios no disponen de envases adecuados para los residuos tipo II que generan. Esta deficiencia es significativamente más acusada (chi ${ }^{2}=16.68, \mathrm{p}<$ 0.001 ) en los hospitales pequeños (en los cuales ocurre en el $88,5 \%$ de los servicios) que en los hospitales grandes (11.5\%). La situación es, sin embargo, diferente en lo que respecta a los residuos tipo III (traumáticos o no) para los que el $100 \%$ de los servicios están dotados de envases adecuados, tanto en los hospitales grandes como en los pequeños. Independientemente del tipo de envase, la ubicación y la accesibilidad fueron buenas en todos los servicios, tanto de hospitales grandes como pequeños.

En cuanto a la clasificación del residuo por parte del personal hospitalario, el residuo clínico o residuo tipo II es, sin duda, el peor clasificado. Para el conjunto de los servicios, este tipo de residuos se elimina en un $82,4 \%$ de las ocasiones en un envase de rango inferior o superior al adecuado. Por otro lado, las diferencias en la clasificación de este residuo entre los servicios de hospitales pequeños y grandes $(85.4 \%$ y $79.0 \%$, respectivamente) no eran estadísticamente significativas.

Para el total de los servicios, el residuo tipo I se clasifica incorrectamente en el $52.7 \%$ de los casos. Este porcentaje era del
74.4\% para los hospitales grandes y del $33.3 \%$ para los pequeños. La diferencia entre ambos era estadísticamente significativa ( chi $\left.^{2}=15.36, \mathrm{p}<0.001\right)$. Mayoritariamente $(98.9 \%)$, los servicios de nuestros hospitales, independientemente de su tamaño clasifican adecuadamente el residuo tipo III o especial.

No se han detectado problemas importantes en cuanto a la recogida y transporte del residuo dentro de cada servicio, pero sí en cuanto a la disponibilidad de depósitos intermedios. En cste sentido, el $66 \%$ del total de servicios no cuentan con esta dependencia. La diferencia entre hospitales grandes y pequeños para esta deficiencia $(53.4 \%$ y $77.0 \%$, respectivamente) era estadísticamente significativa $\left(\mathrm{chi}^{2}=5,62, \mathrm{p}<0,05\right)$. En los casos en que existe, esta dependencia cumple mayoritariamente las características prescritas en la normativa de referencia en Asturias $^{21}$, exceptuando la disponibilidad de un aparato sanitario de vertido que estaba ausente en el $48 \%$ de los casos y de ventilación adecuada que está ausente en el $29 \%$ de los casos.

En la Tabla 2 se muestra el porcentaje de hospitales, según su tamaño, que cumplen las características descritas en el protocolo del Anexo II. Respecto a la disponiblidad y características del depósito general de resíduos, éste cumple las especificaciones normativas $^{21}$ en el $28.5 \%$ de los hospitales pequeños y en ninguno de los hospitales grandes. Con carácter general, la circulación de los residuos por el hospital sólo se realiza mediante circuitos diferenciados, espacial y temporalmente, en un $28,5 \%$ de los hospitales pequeños y en ningún caso en los hospitales grandes.

No hemos hallado diferencias estadísticamente significativas en lo que respecta a la disponibilidad por parte del personal sanitario de información escrita sobre la clasificación y el mancjo de los residuos, a favor de los hospitales grandes $(60 \%)$ frente a los hospitales pequeños (42.8\%). Por otro lado, el Servicio de Medicina Preventiva o su 
Tabla 2

Porcentaje de hospitales, desglosados en «grandes» y «pequeños» que cumplen las características descritas en el protocolo siguiendo el anexo 2

\begin{tabular}{|lcc|}
\hline & $\begin{array}{c}\text { Hospitales grandes } \\
(\%)\end{array}$ & $\begin{array}{c}\text { Hospitales pequeños } \\
\text { (\%) }\end{array}$ \\
\hline Circulación intermedia por circuitos diferenciados & 0,0 & 28,5 \\
Carros de transporte adecuados & 100 & 100 \\
Depósito General del hospital con caracteristicas adecuadas & 0,0 & 28,5 \\
Almacenamiento adecuado en el Depósito General & 0,0 & 28.5 \\
Recogida final con periodicidad adecuada & 100 & 100 \\
Información escrita en la mayoría de los servicios & 60,0 & 42.8 \\
Supervisión adecuada de la gestión de residuos por el Servicio & 40,0 & 57.1 \\
de Preventiva o responsable equivalente & & \\
\hline
\end{tabular}

equivalente, supervisa de manera adecuada todos los aspectos relativos a la gestión de los residuos en el $40 \%$ de los hospitales grandes y en el $57,4 \%$ de los pequeños. Esta diferencia tampoco es estadísticamente significativa.

\section{DISCUSIÓN}

El aspecto más deficitario en la gestión de residuos sanitarios en los hospitales asturianos es, sin duda, la clasificación de los mismos. En este sentido, existen varios factores implicados: no todas las unidades o servicios de nuestros hospitales tienen disponibles, con carácter permanente, envases adecuados a cada tipo de residuos, aunque este déficit no es cuantitativo sino cualitativo. Esto provoca que el personal utilice las bolsas y contenedores de que dispone, aunque no sean los específicos para el tipo de residuo que están desechando. En ocasiones, los encargados de la limpieza no colocan bolsas de cada tipo en cadiı unidad por olvido, o por no disponer de ellas. Otras veces es el propio hospital el que opta por no adquirir un determinado tipo de bolsa en base al protocolo interno de gestión de residuos del centro.

En cualquier caso, aún disponiendo de envases adecuados, no en todos los hospitales existe, entre el personal, una sensibilización y un entrenamiento adecuado para el correcto manejo del residuo. Nuestros hospitales tienen protocolos de gestión interna, pero únicamente en la mitad de ellos sus indicaciones están expuestas, con carácter mayoritario, en los servicios para poder ser consultados por el personal. Por tanto, la información existe pero no se difunde correctamente y los trabajadores no tienen acceso suficiente a ella. En general, se tiende a sobrevalorar la peligrosidad del residuo, tendiendo a clasificarlos en envases de rango superior al correspondiente, lo que traduce la desinformación existente sobre los riesgos reales asociados a cada tipo de residuo (diferencia entre el riesgo percibido y el real ${ }^{24}$. En otros aspectos evaluados, como la recogida y transporte de los residuos a través de la unidad, no se han observado deficiencias.

La mayor parte de los hospitales pequeños carecen de depósitos intermedios. Aparentemente, esto no plantea problemas pues es habitual que generen poca cantidad de residuos y estos pueden ser retirados directamente desde los lugares de producción. Algo más de la mitad de los grandes hospitales poseen en cada planta depósitos intermedios, aunque cuando existen, la mayoría de ellos tienen las características descritas en el Manual de referencia en cuanto a ubicación, limpieza, ventilación, dimensiones, etc.

Sería importante disponer de circuitos diferenciados y montacargas específicos para el transporte de residuos, especialmente en 
los hospitales grandes en que la producción es muy abundante. No obstante, la actual infraestructura de los centros no lo permite.

En ninguno de los grandes hospitales existe un depósito general del tipo de un local cerrado especialmente dedicado a este fin y los residuos permanecen en contenedores al aire libre desde donde son retirados por la empresa de limpieza.

Sólo tres de los doce hospitales estudiados cuentan con servicio de Medicina Preventiva. Nos parece que es muy necesario dotar a nuestros hospitales de este tipo de servicio, que tiene, entre otras, la función de control de la gestión de los residuos de una manera integral.

En general, se presta más atención a los problemas derivados del uso incorrecto de los envases, que a realizar unas prácticas adecuadas para evitar esos problemas. Por ejemplo, todos los centros estudiados tienen protocolos establecidos para actuar ante un pinchazo accidental con aguja. Aunque este aspecto es importante, debería hacerse más énfasis en la prevención primaria de este tipo de accidentes cuya incidencia disminuiría si se usaran correctamente los contenedores ${ }^{25-27}$.

Debemos insistir en la necesidad de una correcta clasificación de los residuos, en origen, por dos motivos. De un lado, el ex- ceso de celo clasificando residuos asimilables a urbanos o clínicos como residuos especiales, se traduce en una sobrecarga económica para el hospital, especialmente en los grandes centros en que el volumen anual de residuos es considerable. Por otro, la opción inversa, es decir la utilización de envases de rango inferior, especialmente para los residuos de tipo III (de alto potencial transmisor de enfermedades por sí mismos), es absolutamente desaconsejable por el riesgo que implica para la salud del personal ${ }^{28-31}$.

Hay una serie de aspectos mejorables a corto plazo en los hospitales asturianos en cuanto a la gestión de los residuos sanitarios: a) asegurar un suministro permanente a todas las unidades y servicios de envases adecuados al tipo de residuos producidos en ellas; b) informar verbalmente y por escrito a todo el personal, sanitario y no sanitario, del uso correcto de los envases; c) asegurar que esta información sea trasmitida periódicamente, especialmente en los peíiodos vacacionales o cuando se produzcan cambios en la plantilla habitual del hospital; d) sensibilizar al personal acerca de la necesidad de una adecuada clasificación del residuo; e) dotar de Servicios de Medicina Preventiva (o al menos de un médico encargado específicamente de csta función) a aqucllos hospitales en que no existe, para establecer un programa de control de la gestión de estos residuos. 


\section{ANEXO 1}

\section{CENTRO SANITARIO:}

\section{UNIDAD/SERVICIO:}

1) ¿Está dotada la Unidad/Servicio de envases adecuados, en cuanto al tipo, a las categorías de residuos producidos?

Tipo I:

Tipo II:

Tipo III:
SI $\square \quad$ NO

SI

SI
$\mathrm{NO}$

$\mathrm{NO}$

2) ¿Está dotada la Unidad/Servicio de envases adecuados al número de residuos producidos?

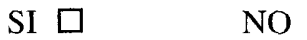

3) ¿La ubicación y accesibilidad de los envases para residuos en la Unidad/Servicio es la adecuada?

\section{SI $\square \quad$ NO}

4) ¿El personal sanitario y no sanitario de la Unidad/Servicio realiza una clasificación adecuada de los residuos y utiliza correctamente los envases para cada tipo de ellos?:

Tipo I:

Tipo II:

Tipo III:
SI $\square \quad$ NO

SI $\square$

SI

NO

NO

5) ¿En la Unidad/Servicio los envases con residuos se recogen con la periodicidad adecuada?

$$
\text { SI } \square \quad \text { NO }
$$

6) ¿El transporte de residuos por la Unidad/Servicio se realiza de manera adecuada?

SI

7) ¿Existe Depósito Intermedio de residuos en la Unidad/Servicio?

SI

NO

En caso afirmativo, ¿reúne éste las características adecuadas?

- Ubicación:

- Ventilación:

- Dimensiones:

- Limpieza:

- Aparato Sanitario:
SI $\square$

SI $\square$

SI $\square$

SI $\square$

SI
NO 
8) ¿Se almacenan los residuos en el Depósito Intermedio de la Unidad/Servicio de una manera adecuada?

SI $\square \quad$ NO

9) ¿La evacuación de residuos del Depósito Intermedio de la Unidad/Servicio se hace con una periodicidad adecuada?

SI $\square \quad$ NO

ANEXO 2

CENTRO SANITARIO:

1) ¿La circulación intermedia del residuo por el hospital se hace con circuitos diferenciados (espacial/temporalmente)?

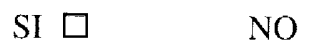

2) ¿Se emplean carros o vehículos de transporte adecuados para los residuos sanitarios?

SI $\square \quad$ NO

3) ¿Reúne el depósito final de residuos del Centro las características adecuadas?

SI $\square \quad$ NO

4) ¿Es adecuado el almacenamiento de los residuos en el depósito final del Centro?

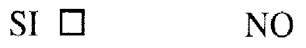

5) ¿La recogida final de residuos por los servicios externos de recogida se hace con una periodicidad adecuada?

SI $\square$

6) ¿Tiene el personal del Centro información escrita con instrucciones sobre la gestión de residuos?

MAYORITARIAMENTE

SI

PARCIALMENTE

NO

$\mathrm{NO}$

7) ¿El Servicio de Medicina Preventiva o el responsable equivalente realiza una supervisión adecuada de la gestión de residuos en el Centro?

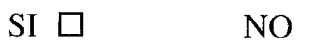




\section{BIBLIOGRAFIAA}

1. OCDE. La gestion des déchets dans les pays Membres de l'OCDE. París: Organisation de Coopération et Developpement Économiques, 1976.

2. Miramontes $H$. Progress in establishing safety protocols based on CDC and OSHA recommendations. Infect Control Hosp Epidemiol 1990; 11(10): $561-562$

3. Aparicio M J. Gestión de los residuos sólidos hospitalarios desde una perspectiva sanitario medio-ambiental. Rev Enferm 1991; 14: 73-76.

4. Berger K J. New priority: pollution risk management. Hospitals 1991; 65(13): 42-44.

5. McCormick R D, Meisch M G, Ircink F G, Maki D G. Epidemiology of hospital sharps injuries: a 14-year prospective study in the pre-AIDS and AIDS eras. Am J Med 1991; 91(3B): 301-307.

6. Larderel A. La politique française en matière de gestion déchets. Techniques Hospitaliers 1978; 390: 90-116.

7. Directiva 89/369/CEE. Reglamento del Consejo del 8.06.1989 sobre prevención de la contaminación del aire causada por nuevos dispositivos de incineración para residuos de conjuntos urbanos.

8. Directiva 91/689/CEE. Directiva del Consejo del 12.12.1991 relativa a los residuos peligrosos.

9. Arcos P. González R. Residuos Sanitarios y Hospitalarios: Un problema a corto plazo. Todo Hospital 1991; 77: 57-61.

10. $\Lambda$ rcos P, González R. Gestión de Residuos Hospitalarios: El modelo del Principado de Asturias. Todo Hospital 1994; 108 : 29-36.

11. Boletín Oficial de Cantabria. Decreto 22/90, de 7 de mayo, por el que se regula la normativa para la gestión de residuos hospitalarios en Cantabria.

12. Boletín Oficial de Cantabria. Decretos $300 / 92$, de 24 de noviembre y $71 / 94$, de 22 de febrero, por los que se regulan la ordenación de la gestión de los residuos sanitarios en Cantabria.

13. Boletín Oficial de la Comunidad Autónoma de Aragón. Decreto 29/1995, de 21 de febrero, sobre gestión de los residuos sanitarios en la Comunidad Autónoma de Aragón.
14. Collins C H. Treatment and disposal of clinical and laboratory waste. Med Lab Sci 1991; 48(4): 324-331.

15. McVeigh P. OR nursing and environmental ethics. Medical waste reduction, reuse, and recycling. Todays OR Nurse 1993; 15 (1) : 13-18.

16. Halbwachs H. Solid waste disposal in district health facilities. World Health Forum 1994; 15: 363-367.

17. Consorcio para la Gestión de los Residuos Sólidos en Asturias. Resumen Anual de Residuos Hospitalarios 1995. Oviedo: Consorcio para la Gestión de los Residuos Sólidos en Asturias, 1995.

18. Bencko V, Culikova H. Hospital Waste Management Practice in the Czech Republic. Cent Eur Public IIealth 1993; 1(1) : 57-59.

19. Koska M T, Anderson la J, Hudson T, Johnsson J, Hard R, Eubanks P. 12 cost-cutting measures. Hospitals use creative ideas to again control of expenses. Hospitals 1992; 66 (22): 24-29.

20. Catálogo Nacional de Hospitales. Madrid: Ministerio de Sanidad y Consumo, 1992.

21. Arcos P, Bances D. La Gestión de los Residuos Sanitarios. 2. ${ }^{\mathrm{a}}$ ed. Oviedo: Servicio Central de Publicaciones del Principado de Asturias, 1994.

22. Joint Commission on Accreditation of Health Care Organizations. A.M.H. Accreditation Manual for Hospitals, 1992. Vol I-II. Oakbrook (USA): Joint Commision on Accreditation of Health Care Organizations, 1992.

23. Proyecto del Real Decreto por el que se regulan las condiciones para la gestión de residuos procedentes de establecimientos sanitarios. Documento Ref: 20- SR/PP. Ministerio de Sanidad y Consumo. Dirección General de Salud Pública, 1995

24. Keene J H. Medical waste: a minimal hazard. Infect Control Hosp Epidemiol 1991; 12 (11): 682685 .

25. Adegboye A A, Moss G B, Soyinka F, Kreiss J K. The epidemiology of needlestick and sharp instruments accidents in a Nigerian hospital. Infect Control Hosp Epidemiol 1994; 15(1): 27-31.

26. Hedrick C. A process for selecting a safety needle system in a community teaching hospital. J Intraven Nurs. 1993; 16(5): 299-302.

27. Makofsky D, Cone J E. Installing needle disposal boxes closer to the bedside reduces needle-recapping rates in hospital units. Infect Control Hosp Epidemiol 1993; 14(3): 140-144. 
28. Moss S T, Clark R F, Guss D A, Rosen P. The managenent of sharps in the emergency department: is it safe? J Emerg Med 1994; 12: 745752.

29. Gould D. Sharps handling and disposal: a study. Nurs Stand 1994; 8: 25-28.
30. Tan R, Noble M A. Sharps utilization and disposal in British Columbia Physicians offices. Can J Public Health 1993; 84: 31-34.

31. Burns J. Safety first: the key consideration. Mandgament of clinical waste. Prof Nurse 1991; 7(3): 183-187. 